ORIGINAL ARTICLE

\title{
Urinary bisphenol A and plasma hormone concentrations in male workers exposed to bisphenol A diglycidyl ether and mixed organic solvents
}

\author{
T Hanaoka, N Kawamura, K Hara, S Tsugane
}

Occup Environ Med 2002;59:625-628

See end of article for authors' affiliations

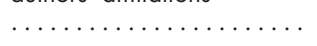

Correspondence to: Dr T Hanaoka,

Epidemiology and

Biostatistics Division,

National Cancer Center

Research Institute East,

6-5-1 Kashiwanoha, Chiba

277-8577, Japan;

thanaoka@east.ncc.go.jp

Accepted 12 March 2002

\begin{abstract}
Aims: To evaluate effects of exposure to bisphenol A diglycidyl ether (BADGE) on urinary excretion of bisphenol A, and plasma gonadotrophic hormones and testosterone in male epoxy resin sprayers. Methods: Cross sectional study of 42 workers whose job was to spray epoxy resin hardening agents including BADGE and mixed organic solvents, and 42 matched control workers without BADGE use in the same machine plants.

Results: Concentrations of urinary bisphenol A were higher in the epoxy resin sprayers (median 1.06 $\mathrm{\mu mol} / \mathrm{mol}$ creatinine) compared with the controls (median $0.52 \mu \mathrm{mol} / \mathrm{mol}$ creatinine). Urinary metabolite concentrations of organic solvents used were all higher in the epoxy resin workers compared with the controls. Endocrinological examination showed different concentrations of follicle stimulating hormone (FSH) between the epoxy sprayers (median $5.3 \mathrm{mlU} / \mathrm{ml}$ ) and the controls (median $7.6 \mathrm{mlU} / \mathrm{ml}$ ). FSH showed a mild correlation with urinary bisphenol A, but not with the metabolites of organic solvents. Luteinising hormone and free testosterone concentrations did not differ between the two groups. Conclusion: BADGE may generate bisphenol A endogenously. Results suggest that bisphenol A may disrupt secretion of gonadotrophic hormones in men. The clinical significance of endocrine disrupting effects by bisphenol A should be further investigated in male workers exposed to bisphenol $\mathrm{A}$.
\end{abstract}

$\mathrm{B}$ isphenol A diglycidyl ether (BADGE) is a member of the glycidyl ether group. Glycidyl ethers have been widely used as basic components of epoxy resins since the late 1940s. BADGE and its oligomers are major components of epoxy resins. Occupational exposure to BADGE occurs in its production, epoxy resin production, and various uses of epoxy products. ${ }^{1}$ Apart from allergic reaction, ${ }^{2-5}$ its health effects and metabolism in humans have rarely been reported. An oestrogen receptor binding capacity of BADGE was reported to be low in a previous in vitro study. ${ }^{6}$ However, if bisphenol A is generated from BADGE through a metabolic transformation in the body, the endogenous bisphenol A may affect endocrinological function. In mice orally exposed to BADGE, the amount of bisphenol A excretion was reported to be low, but no observations have been reported in exposed humans. Animal experiments have shown effects of bisphenol A on male reproductive function or generative organs, ${ }^{7-9}$ but no effects have been reported in men.

To evaluate the effects of occupational exposure to BADGE on urinary excretion of bisphenol A, and the effects of endogenous bisphenol A on plasma gonadotrophic hormones and testosterone in male workers, we conducted a cross sectional

\section{Main messages}

- BADGE may generate bisphenol A endogenously.

- Bisphenol A may disrupt secretion of gonadotrophic hormones in men.

\section{Policy implication}

- Clinical significance of endocrine disrupting effects by bisphenol A should be further investigated in male workers exposed to bisphenol A. study of epoxy resin workers who sprayed BADGE with mixed organic solvents in plastic plants. Their urinary bisphenol A and plasma hormones (including luteinising hormone (LH), follicle stimulating hormone (FSH), and free testosterone) were examined.

\section{SUBJECTS AND METHODS}

\section{Subjects and sample collection}

Forty two male workers, whose work was to spray BADGE with mixed organic solvents as an epoxy resin hardening agent in a machine plant and two related plants located in Aichi Prefecture, served as the exposed workers in this study. They included all workers using BADGE in these plants. According to the product specification sheets of seven hardening agents used, they contained 10-30\% BADGE. The product sheets indicated that these agents also contained toluene $(0-30 \%)$, xylene $(0-20 \%), \quad 2$-ethoxyethanol (0-20\%), 2-butoxyethanol (0-20\%), and methyl isobutyl ketone (0$30 \%)$. The workers used the above agents in spray rooms which were not completely airtight. They were in charge of the spraying at least three hours a day, and wore protection devices during the spraying, but their workplace atmosphere was polluted.

To select control subjects, 82 male workers whose age was similar to that of the epoxy resin workers, were randomly selected from 1202 assembly workers who did not use BADGE in the same plants. None refused to be recruited for this study. Forty two workers were finally selected as the controls to be matched to each epoxy resin worker by age $( \pm 3)$ and number of cigarettes/day.

Abbreviations: $B A D G E$, bisphenol A diglycidyl ether; FSH, follicle stimulating hormone; $\mathrm{LH}$, luteinising hormone; HPLC, high performance liquid chromatography; $E C D$, electrochemical detector; $B D L$, below detection limit 
This study was conducted according to the Declaration of Helsinki. All subjects participated in the study as volunteers, and gave their written informed consent.

Mean age $(37 \pm 9,38 \pm 10)$, percentage of current smokers $(86 \%, 86 \%)$, and number of cigarettes/day among smokers $(21 \pm 7,21 \pm 6)$ were comparable between the epoxy resin sprayers and the controls. The percentage of alcohol drinkers was significantly lower in the epoxy resin sprayers (43\%) than in the controls $(57 \%)(p=0.031)$.

Urine and blood samples were collected at periodic health examinations conducted in June and July, 1999, but not after a night shift. Urine samples were collected in the morning (10-12 am), but not on the first day of the week. Peripheral blood was collected in an EDTA-2Na tube at 10-12 am on the same day as urine sampling. Plasma was collected by centrifugation. A simple questionnaire was used to obtain lifestyle information, including smoking and alcohol drinking habits.

\section{Analytical methods}

Urinary bisphenol A was measured by high performance liquid chromatography (HPLC) with an electrochemical detector (ECD, Model 5600A CoulArrey Detector, ESA, Inc., Chelmsford, MA) using modifications of methods reported previously. ${ }^{10} 11$ Briefly, $50 \mu \mathrm{l}$ of $\beta$ glucuronidase (Wako Chemicals, Osaka, Japan) and $200 \mu \mathrm{l}$ of $0.1 \mathrm{M}$ sodium acetate buffer (pH 5.0, 0.1\% ascorbic acid, $0.01 \%$ EDTA) were added to $200 \mu$ l of urine. Dimethylbutylidene bisphenol (Wako Chemicals) was added to this solution as an internal standard. After incubation at $37^{\circ} \mathrm{C}$ for three hours, $1.2 \mathrm{ml}$ ethanol was added to the solution. One $\mathrm{ml}$ of supernatant was obtained by centrifugation at $12000 \mathrm{~g}$ for 15 minutes, and was evaporated by a vacuum evaporator. Residue was resolved by $0.3 \mathrm{ml}$ of $50 \%$ methanol. After filtration, 40 $\mu \mathrm{l}$ of the solution was injected into the HPLC system. The analytical conditions of the HPLC-ECD system were as follows: octadecil column (ODS250, 250×4.6 mm, MC Medical, Inc., Tokyo, Japan); mobile phase (A), acetonitrile-water-phosphoric acid (20:79.8:0.2); mobile phase (B), acetonitrile-waterphosphoric acid (80:18.8:0.2); linear gradient programme, 25\%B (0-28 min), 25\%B-100\%B (28-37 min); ECD detector voltages, 360, 400, 440, 480, 520, 560, 600, and $640 \mathrm{mV}$. The peak of bisphenol A was confirmed using profiles of reactions in these eight channels. The bisphenol A detection limit in this system was approximately $10 \mathrm{pg}(0.05 \mathrm{pmol})$. The bisphenol A concentration was determined by a linear regression line of standard bisphenol A (Tokyo Kasei Co., Tokyo, Japan), and was adjusted by a recovery rate of the internal standard. The recovery rates of bisphenol A and the internal standard were approximately $100 \%$. The coefficient of variation of the measure was $<10 \%$. A blank sample containing water (Milli-Q SP VOC, Millipore Co., MA) instead of urine was treated using the same method, and analysed. The value of the blank sample $(0.5$ $\mathrm{pmol} / \mathrm{ml}$ ) was subtracted from the values of the samples. If the value of a sample was below the blank level, the sample level was considered "not detected". The water was confirmed to contain no bisphenol A. The bisphenol A concentration was adjusted by urinary creatinine concentration.

We analysed the concentrations of the following urinary metabolites: $o, m, p$-cresol, $o, m, p$-methylhippuric acid, 2-ethoxyacetic acid, 2-butoxyacetic acid, and methyl isobutyl ketone.

Urinary $o$-cresol was measured using the method of Taguchi and colleagues. ${ }^{12}$ Briefly, the hydrolysed sample was extracted with ethyl acetate. The extracted analyte was measured using a high performance liquid chromatograph (HPLC, Model L-7000, Hitachi Ltd, Ibaraki, Japan) equipped with a fluorescence spectrophotometer (Ex270/Em290 nm, Model F-1050, Hitachi Ltd).

The methylhippuric acid concentration was determined according to Hasegawa and colleagues. ${ }^{13}$ Briefly, a diluted urine sample was measured using an HPLC (Model L-7000, Hitachi Ltd) with UV detector (230 nm, Model L-4000, Hitachi Ltd).

Urinary 2-ethoxyacetic acid and 2-butoxyacetic acid were measured according to the method of Smallwood and colleagues. ${ }^{14}$ Briefly, a urine sample was extracted by methylene chloride. The methylene chloride layer was injected into a gas chromatograph (GC) equipped with a flame ionisation detector (Model G-3000, Hitachi Ltd).

Methyl isobuthyl ketone in urine was analysed by the head space method. Briefly, urine was heated, then the head space air inside the vial was injected into a GC equipped with a flame ionization detector (Hitachi Model 263-50, Hitachi Ltd).

Detection limits were as follows: cresol $4 \mathrm{ng} / \mathrm{ml}$; methylhippuric acid $1 \mu \mathrm{g} / \mathrm{ml}$; 2-methoxyacetic acid $2 \mu \mathrm{g} / \mathrm{ml}$; and 2-butoxyacetic $1 \mu \mathrm{g} / \mathrm{ml}$.

Luteinising hormone (LH), follicle stimulating hormone (FSH), and free testosterone in plasma were measured by radio immunosolvent assay in a commercial laboratory (SRL Inc., Tokyo, Japan). The reference values for these determinations provided by the laboratory were $1.8-5.2 \mathrm{IU} / \mathrm{ml}, 2.9-8.2$ $\mathrm{IU} / \mathrm{ml}$, and $14-40 \mathrm{pg} / \mathrm{ml}$, respectively. The coefficients of variation for these measures were $<5 \%$.

\section{Statistical analysis}

Values, including urinary bisphenol A, metabolites, and plasma hormones, were log transformed for statistical analysis because their distribution was not normal. Differences between the two groups (BADGE workers and controls) were tested by paired $t$ test. Pearson's correlation coefficient was calculated to assess the degrees of relations between the metabolites and plasma hormones. To adjust for age and alcohol drinking habits, possible confounders, a multiple linear regression analysis was used to assess relations between the metabolites and the hormones. Differences in proportions concerning basic characteristics between BADGE workers and controls were tested by the $\chi^{2}$ method. A p value less than 0.05 (two tailed) was considered significant.

\section{RESULTS}

Urinary metabolites of organic solvents were detected more frequently in the epoxy resin workers compared with the controls: $o$-cresol $(81 \%, 33 \%)$, methylhippuric acids $(62 \%, 12 \%)$, and 2 -butoxyacetic acid $(62 \%, 0 \%)$. Median concentrations (range) were as follows: 0 -cresol, 33 (below detection limit (BDL) to 816) $\mu \mathrm{mol} / \mathrm{mol}$ creatinine; methylhippuric acids, 1.7 (BDL to 23) $\mu \mathrm{mol} / \mathrm{mol}$ creatinine, and 2-butoxyacetic acid, 0.6 (BDL to 70) $\mathrm{mmol} / \mathrm{mol}$ creatinine in the epoxy resin sprayers. In controls, $o$-cresol was BDL (BDL to 467) $\mu \mathrm{mol} / \mathrm{mol}$ creatinine and methylhippuric acids were BDL (BDL to 4) $\mu \mathrm{mol} / \mathrm{mol}$ creatinine. Concentrations of urinary 2-butoxyacetic acid in the controls were below the detection limit. Urinary metabolite concentrations were all higher in the epoxy resin workers compared with the controls. Urinary 2-ethoxyacetic acid and MIBK were not detected in either group.

Figure 1 shows the concentrations of bisphenol A in urine. A significant difference in bisphenol A concentrations was observed between the epoxy resin sprayers (median 1.06; range: not detected to $11.2 \mu \mathrm{mol} / \mathrm{mol}$ creatinine) and the controls (median 0.52; range: not detected to $11.0 \mu \mathrm{mol} / \mathrm{mol}$ creatinine $)(\mathrm{p}=0.002$, average difference $=2.5 ; 95 \%$ confidence interval (CI) 1.4 to 4.7). We could not detect bisphenol A for three epoxy resin sprayers and one control.

Table 1 shows the average plasma hormone concentrations. Free testosterone concentrations did not differ between the epoxy resin sprayers and controls $(\mathrm{p}=0.74$, average difference $=1.0 ; 95 \% \mathrm{CI}-1.1$ to 1.1$)$. No difference in $\mathrm{LH}$ concentrations between the two groups was observed $(\mathrm{p}=0.41$, average difference $=1.1 ; 95 \% \mathrm{CI}-1.1$ to 1.3$)$. However, we observed a significant difference in FSH concentrations between the sprayers (median $5.3 \mathrm{mIU} / \mathrm{ml}$ ) and the controls $($ median $7.6 \mathrm{mIU} / \mathrm{ml})(\mathrm{p}=0.022$, average difference $=$ $-1.3 ; 95 \% \mathrm{CI}-1.5$ to -1.0$)$.

FSH showed a mild correlation with urinary bisphenol A (correlation coefficient $-0.20, p=0.071$, fig 2 ), but not with 


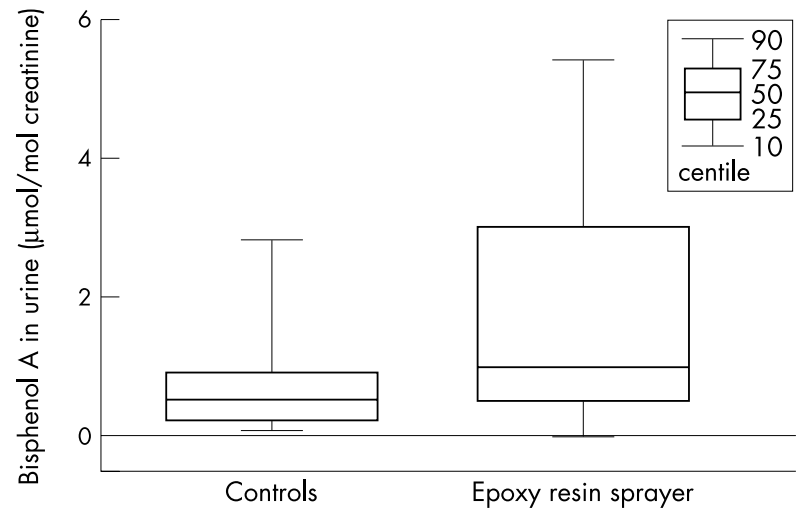

Figure 1 Concentrations of bisphenol $A$ in urine of epoxy resin sprayers who used bisphenol A diglycidyl ether $(n=42)$ and the control workers $(n=42)$. The difference between the two groups was statistically significant $(p=0.002)$.

Table 1 Median (interquartile range) of plasma hormone levels in workers using bisphenol A diglycidyl ether and control workers

\begin{tabular}{lcl}
\hline Hormones & $\begin{array}{l}\text { Exposed sprayers } \\
(\mathrm{n}=42)\end{array}$ & $\begin{array}{l}\text { Control workers } \\
(\mathrm{n}=42)\end{array}$ \\
\hline $\mathrm{LH}(\mathrm{mlU} / \mathrm{ml})$ & $4.0(4.0-5.0)$ & $4.0(3.0-6.0)$ \\
$\mathrm{FSH}(\mathrm{mlU} / \mathrm{ml})$ & $5.3(4.0-8.3)$ & $7.6(5.4-11.0)$ \\
Free testosterone $(\mathrm{pg} / \mathrm{ml})$ & $15.2(12.7-18.7)$ & $15.1(12.5-17.1)$ \\
\hline
\end{tabular}

the metabolites of organic solvents. The multivariate analysis showed a statistically significant relation between FSH and bisphenol A, adjusted for alcohol drinking habits $(\mathrm{p}=0.045)$. No significant correlations were observed between other hormones and urinary metabolites (table 2).

\section{DISCUSSION}

In this cross sectional study, we observed an increased excretion of bisphenol A and decreased plasma FSH in the male workers who sprayed epoxy resin hardening agents containing BADGE compared to the matched controls.

A previous in vivo study reported many types of urinary metabolites in mice which had orally ingested BADGE. ${ }^{15}$ According to the study, bisphenol A was one of the minor metabolites, and the amount was reported to be small. ${ }^{16}$ The experiment by Climie et al used radiolabelled BADGE, so the results of their study seemed to be generally reliable. Nevertheless, we observed an increased mean concentration of bisphenol A in the exposed sprayers.

In this study, we could not analyse major metabolites of BADGE, for example, bis-diol of BADGE, ${ }^{15}$ because their

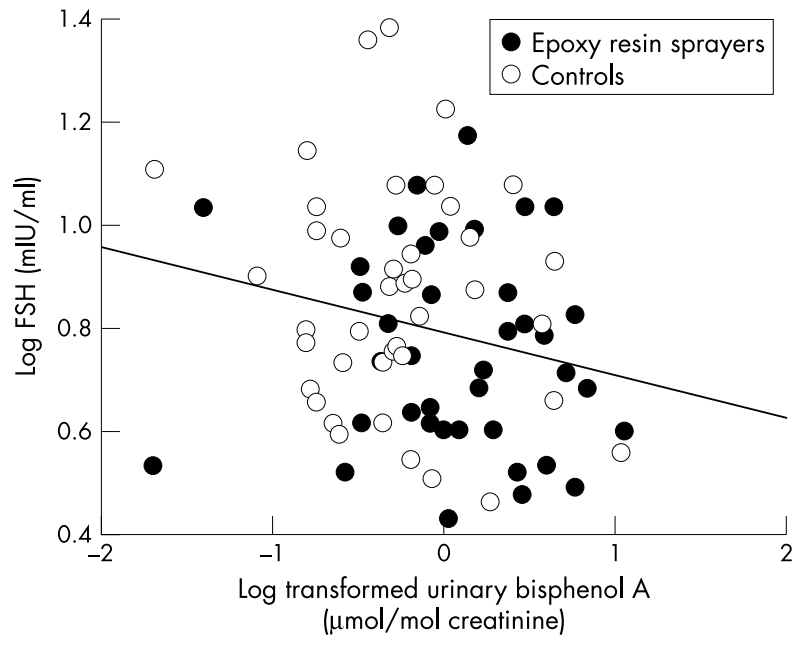

Figure 2 Relation between urinary bisphenol A concentrations and plasma FSH in all subjects $(\mathrm{n}=84$, correlation coefficient -0.20 , $p=0.071)$.

standard reagents were not available. Thus, we could not directly confirm exposure to BADGE in the sprayers. However, increased metabolites of some organic solvents in the epoxy resin hardening agents supported the hypothesis that BADGE also entered their body during work. The vapour pressure of BADGE is unknown, but as it is generally used as a liquid, workers can intake the mist. Consumption of canned beverages, a suspected potential source of bisphenol A exposure in daily life, ${ }^{17}$ was comparable between the epoxy resin sprayers and the matched controls, but few subjects consumed them in either group. Urinary bisphenol A was detected in most of the controls, therefore other unknown sources of bisphenol A intake cannot be excluded. However, no specific source contributed to bisphenol other than in BADGE in the sprayers, because a study of urinary bisphenol A concentrations in rural residents in Japan has shown that most of the general population have concentrations similar to those of the controls in the present study (manuscript in preparation). It is reasonable to consider that bisphenol A was generated endogenously by occupational exposure to BADGE.

One control showed a high concentration of bisphenol A as shown in fig 1 . One possibility is that he was exposed to BADGE passively near the spraying works. Similar phenomena were observed in urinary metabolites of organic solvents. Use of protective devices and passive exposure might cause a selection bias in this survey.

Effects of bisphenol A on the endocrinological system have been reported. Its oestrogen receptor binding capacity has been observed in earlier reports. ${ }^{18}{ }^{19}$ Concerning genital function in males, vom Saal et al observed decreased sperm production, although a negative result has also been reported, ${ }^{20}$ and Takao $e t$

Table 2 Standardised partial regression coefficients between selected serum hormones and urinary metabolites in spray workers and controls

\begin{tabular}{|c|c|c|c|c|c|c|}
\hline \multirow[b]{3}{*}{ Metabolites } & \multicolumn{6}{|c|}{ Hormones } \\
\hline & \multicolumn{2}{|c|}{$\mathrm{LH}(\mathrm{mlU} / \mathrm{ml})$} & \multicolumn{2}{|c|}{$\mathrm{FSH}(\mathrm{mlU} / \mathrm{ml})$} & \multicolumn{2}{|c|}{ Free testosterone $(\mathrm{pg} / \mathrm{ml})$} \\
\hline & $r$ & $\mathrm{p}$ & $r$ & $\mathrm{p}$ & $r$ & $\mathrm{p}$ \\
\hline Bisphenol A ( $\mu \mathrm{mol} / \mathrm{mol}$ creatinine) & -0.05 & 0.65 & -0.23 & 0.045 & -0.15 & 0.17 \\
\hline o-Cresol ( $\mu \mathrm{mol} / \mathrm{mol}$ creatinine) & -0.05 & 0.64 & -0.03 & 0.82 & -0.13 & 0.23 \\
\hline Methylhippuric acids* ( $\mu \mathrm{mol} / \mathrm{mol}$ creatinine) & -0.01 & 0.90 & -0.07 & 0.56 & 0.19 & 0.08 \\
\hline 2-butoxyacetic acid* $(\mathrm{mmol} / \mathrm{mol}$ creatinine) & -0.02 & 0.88 & -0.18 & 0.12 & -0.06 & 0.61 \\
\hline
\end{tabular}

Log translated values were used for metabolites and hormone levels. Standardised partial regression coefficients were adjusted for age and alcohol drinking habits.

*Half the detection limit was used for a subject whose urinary metabolite was not detected. 
al observed decreased free testosterone and LH in male mice exposed to bisphenol A. ${ }^{8}$ Furthermore, an experiment by Gupta et al showed that bisphenol A decreased epididymal weight in male offspring following maternal exposure. ${ }^{9}$ In men, LH and FSH are involved in sperm production and androgen synthesis. In the present study, LH and free testosterone were not different between the exposed sprayers and the controls, but we observed decreased FSH in the former. The controls in this study were clinically healthy persons confirmed by a periodical health examination. The reference values for FSH determination also supported the fact that they were clinically normal although some showed relatively high concentrations.

In endocrinological studies, timing of blood sampling in the daytime is an important factor for estimation of hormone concentrations. A "cross sectional" evaluation is a major limitation of our study. However, in this study, the timing of blood sampling was almost similar, and was not after the night shift, for all subjects; a previous study observed that diurnal rhythm for FSH was less notable than that for LH and testosterone in men. ${ }^{21}$ This may explain why there was no observable relation between LH and bisphenol A.

We speculate that endogenous bisphenol A bound to oestrogen receptors in the pituitary body, and FSH secretion was directly suppressed. Oestrogen receptors have been found in the human pituitary body, ${ }^{22}$ and Finkelstein et al showed that oestradiol directly inhibited gonadotrophin secretion at the pituitary level in men. ${ }^{23}$ On the other hand, absence of a feedback effect of testosterone on FSH in men has been reported, ${ }^{24}$ a finding that may also support our speculation.

Effects of chemical exposure on male reproduction have been a concern from the late 1800s in occupational medicine. Some organic solvents have been reported to be potential modifiers of male reproductive function. ${ }^{25}$ Morck et al observed increased FSH concentrations in male workers exposed to toluene. ${ }^{26}$ In the present study, BADGE was used with organic solvents. Thus, one possibility is that mixed organic solvents contained in the epoxy resin hardening agents decreased FSH concentrations. The decreased FSH is believed to be caused by effects on the central nervous system, because such effects of organic solvents are well known. However, levels of exposure to the organic solvents were relatively low. It cannot reasonably be assumed that the effects of organic solvent exposure are causative.

Recently, some chemicals have been noted as endocrine disrupting agents. In this context, environmental effects on male fertility have been discussed elsewhere. ${ }^{27}$ According to our observation, serum gonadotrophic hormones may be a sensitive marker for evaluation of the effects of endocrine disrupting chemicals in men. In a previous study, decreased free testosterone was observed in mice, ${ }^{8}$ but we observed no difference between the sprayers and the controls. The difference of FSH observed in the present study occurred within clinically normal conditions. Thus, the clinical significance is still unclear.

\section{Conclusions}

- BADGE may generate bisphenol A endogenously.

- Bisphenol A may disrupt secretion of gonadotrophic hormones in men.

- Clinical significance of the endocrine disrupting effects of bisphenol A should be further investigated in male workers exposed to bisphenol A.

\section{ACKNOWLEDGEMENTS}

We are grateful to Hiroko Hashimoto for her technical assistance. This work was supported in part by a Grant-in-Aid for Research on Environmental Health from the Ministry of Health and Welfare of Japan, and a Grant-in-Aid for Scientific Research from the Ministry of Education, Science, Sports and Culture, Japan.

\section{Authors' affiliations}

T Hanaoka, N Kawamura, S Tsugane, Epidemiology and Biostatistics Division, National Cancer Center Research Institute East, 6-5-1
Kashiwanoha, Chiba 277-8577, Japan

K Hara, Institute for Science of Labour, Japan

\section{REFERENCES}

1 International Agency for Research on Cancer (IARC). Bisphenol A diglycidyl ether. In: IARC Monographs on Evaluation of Carcinogenic Risks in Humans, Vol.71. Lyon: IARC, 1999:1285-9.

2 Kanerva L, Jolanki $R$, Tupasela $O$, et al. Immediate and delayed allergy from epoxy resins based on diglycidyl ether of bisphenol A. Scand J Work Environ Health 1991;17:208-15.

3 Jolanki R, Kanerva L, Estlander T. Allergic patch test reaction to diglycidyl ether of bisphenol $A$ in hardened nail base and top coat. Contact Dermatitis 1996;35:246-7

4 Kanerva L. Occupational allergic contact dermatitis from epoxy resin in a dental nurse with primary sensitization during cyclosporine treatment. Acta Derm Venereol 1996:76:89-90.

5 Kanerva L, Estlander T, Keskinen $\mathrm{H}$, et al. Occupational allergic airbone contact dermatitis and delayed bronchial asthma from epoxy resin revealed by bronchial provocation test. Eur J Dermatol 2000;10:475-7.

6 Olea N, Pulgar R, Perez P, et al. Estrogenicity of resin-based composites and sealants used in dentistry. Environ Health Perspect 1996; 104:298-305.

7 vom Saal FS, Cooke PS, Buchanan DL, et al. A physiologically based approach to the study of bisphenol A and other estrogenic chemicals on the size of reproductive organs, daily sperm production, and behavior. Toxicol Ind Health 1998;14:239-60.

8 Takao T, Nanamiya W, Nagano I, et al. Exposure with the environmental estrogen bisphenol A disrupts the male reproductive tract in young mice. Life Sci 1999;65:2351-7.

9 Gupta C. Reproductive malformation of the male offspring following maternal exposure to estrogenic chemicals. Proc Soc Exp Biol Med 2000;224:61-8

10 Sajiki J, Takahashi K, Yonekubo J. Sensitive method for the determination of bisphenol-A in serum using two systems of high-performance liquid chromatography. J Chromatogr B Biomed Sci Appl 1999;736:255-61

11 Gamache PH, Acworth IN. Analysis of phytoestrogens and polyphenols in plasma, tissue, and urine using HPLC with coulometric array detection. Proc Soc Exp Biol Med 1998:217:274-80.

12 Taguchi T, Horike T, Ogata M. Simultaneous microdetermination of urinary cresol isomers by high performance liquid chromatography [in Japanese]. Medicine and Biology 1993;126:187-191.

13 Hasegawa K, Shiojima S, Koizumi A, et al. Hippuric acid and o-cresol in the urine of workers exposed to toluene. Int Arch Occup Environ Health Perspect 1983;52:197-208.

14 Smallwood A, Debord K, Burg J, et al. Determination of urinary 2-ethoxyacetic acid as an indicator of occupational exposure to 2-ethoxyethanol. Appl Ind Hyg 1988;3:47-50.

15 Climie IJ, Hutson DH, Stoydin G. Metabolism of the epoxy resin component 2,2-bis[4-(2,3-epoxypropoxy)phenyl]propane, the diglycidyl ether of bisphenol A (DGEBPA) in the mouse. Part II. Identification of metabolites in urine and faeces following a single oral dose of 14C-DGEBPA. Xenobiotica 1981;11:401-24.

16 Hutson DH. Bisphenol A diglycidyl ether as a potential metabolic source of bisphenol A [letter; comment]. Environ Health Perspect 1998; 106:A472-3

17 Brotons JA, Olea-Serrano MF, Villalobos M, et al. Xenoestrogens released from lacquer coatings in food cans. Environ Health Perspect 1995:103:608-12.

18 Nagel SC, vom Saal FS, Thayer KA, et al. Relative binding affinity-serum modified access (RBA-SMA) assay predicts the relative in vivo bioactivity of the xenoestrogens bisphenol A and octylphenol. Environ Health Perspect 1997; 105:70-6.

19 Krishnan AV, Stathis P, Permuth SF, et al. Bisphenol-A: an estrogenic substance is released from polycarbonate flasks during autoclaving. Endocrinology 1993;132:2279-86.

20 Ashby J, Tinwell H, Haseman J. Lack of effects for low dose levels of bisphenol $A$ and diethylstilbestrol on the prostate gland of CF1 mice exposed in utero. Regul Toxicol Pharmacol 1999;30:156-66.

21 Albertsson-Wikland K, Rosberg S, Lannering B, et al. Twenty-four-hour profiles of luteinizing hormone, follicle-stimulating hormone, testosterone, and estradiol levels: a semilongitudinal study throughout puberty in healthy boys. J Clin Endocrinol Metab 1997:82:541-9.

22 Pelletier G. Localization of androgen and estrogen receptors in rat and primate tissues. Histol Histopathol 2000;15:1261-70.

23 Finkelstein JS, O'Dea LS, Whitcomb RW, et al. Sex steroid control of gonadotropin secretion in the human male. II. Effects of estradio administration in normal and gonadotropin- releasing hormone-deficient men. J Clin Endocrinol Metab 1991:73:621-8.

24 Hayes FJ, DeCruz S, Seminara SB, et al. Differential regulation of gonadotropin secretion by testosterone in the human male: absence of a negative feedback effect of testosterone on follicle-stimulating hormone secretion. J Clin Endocrinol Metab 2001:86:53-8.

25 Schrader SM, Kanitz MH. Occupational hazards to male reproduction. Occup Med 1994;9:405-14.

26 Morck HI, Winkel P, Gyntelberg F. Health effects of tolvene exposure. Dan Med Bull 1988;35:196-200.

27 Sinawat S. The environmental impact on male fertility. J Med Assoc Tha 2000;83:880-5. 